\title{
A Meta-Study of Qualitative Research Into the Experience of 'Symptoms' and 'Having a Diagnosis' for People Who Have Been Given a Diagnosis of Bipolar Disorder
}

Leo Russell, Duncan Moss

\section{Note}

A corrected version is available in EJOP, Vol. 9 Issue 3 (2013).

In the following, original version, some section headings have not been processed correctly during production. 


\title{
Literature Reviews
}

\section{A Meta-Study of Qualitative Research Into the Experience of 'Symptoms' and 'Having a Diagnosis' for People Who Have Been Given a Diagnosis of Bipolar Disorder}

\author{
Leo Russell ${ }^{\star a}$, Duncan Moss ${ }^{b}$
}

[a] Clinical Psychology Service, Somerset Partnership NHS Foundation Trust, Taunton, United Kingdom. [b] Department of Clinical Psychology, University of Plymouth, Plymouth, United Kingdom.

\begin{abstract}
The purpose of this study is to review the current state of the literature reporting qualitative studies that depict the experiences of 'symptoms' and 'having a diagnosis' for people who have been given a diagnosis of bipolar disorder. The method attempted to combine empirical and discursive approaches and was strongly influenced by guidance from Paterson, Thorne, Canam, and Jillings (2001) on conducting a meta-review. Meta-data analysis was used to compare the studies and, subsequently, nine common themes emerged: 'struggles with identity', 'loss of control', 'disruption, uncertainty and instability', 'negative impact of symptoms across life and the experience of loss', 'negative view of self', 'positive or desirable aspects of mania', 'struggling with the meaning of diagnosis', 'stigma', and 'acceptance and hope'. The meta-method explored and evaluated the qualitative methods that have been used to study this phenomenon, and the meta-theory considered the theoretical underpinnings and contributions of this research. The review concludes that an awareness of these themes could support clinical work with service-users and inform the development of relevant interventions such as interpersonal social rhythm therapy, cognitive behavioural therapy and mindfulness-based cognitive therapy. Further qualitative research is recommended to extend this literature base and include a greater representation of men and people living in non-westernised countries.
\end{abstract}

Keywords: bipolar disorder, diagnosis, symptoms, qualitative, meta-study, mania, review

Europe's Journal of Psychology, 2013, Vol. 9(2), 385-405, doi:10.5964/ejop.v9i2.560

Received: 2012-12-19. Accepted: 2013-04-15. Published: 2013-05-31.

${ }^{*}$ Corresponding author at: Clinical Psychology Service, Foundation House, Wellspring Road, Taunton, TA2 7PQ, England. E-mail: leo.russell@nhs.net

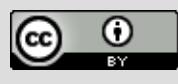

This is an open access article distributed under the terms of the Creative Commons Attribution License

(http://creativecommons.org/licenses/by/3.0), which permits unrestricted use, distribution, and reproduction in any medium, provided the original work is properly cited.

\section{Introduction}

This study seeks to develop ways of understanding the experience of 'symptoms' associated with a diagnosis of bipolar disorder and of the experience of diagnosis itself. The use of this diagnosis as a reference point is a practical solution to the problem of identifying literature that included participants with similar kinds of distress and experiences that are perceived to be unusual. However, as is discussed throughout this review, it is also a problematic solution as diagnostic systems and the application of diagnosis are contentious areas of questionable validity.

\section{Background}

Bipolar Disorder as a Psychiatric Diagnosis - Bipolar disorder is a commonly applied diagnosis of a mental health problem with prevalence rates between 1-1.5\% (Jones, 2004). According to the American Psychiatric As- 
sociation (1994), the criteria for a diagnosis of bipolar disorder require the presence of either 'mania' or 'hypomania' alongside an experience of 'clinical depression'. Unlike many other diagnoses, bipolar disorder appears to have no significant differences in prevalence rates across cultures, age groups or gender compared to culture, age and gender in the general population (Newman, Leahy, Beck, Reilly-Harrington, \& Gyulai, 2001).

People who have been labelled with this diagnosis report significant distress and suffering, poor quality of life, high levels of self-harm and approximately a third of these people attempt suicide at some point (Jones, 2004; Jones \& Bentall, 2006). The diagnosis is also associated with non-adherence to psychological interventions and medication and this is seen as negatively impacting the prognosis (Gaudiano, Weinstock, \& Miller, 2008). However, it should be noted that this epidemiological data is largely based on information gathered within westernised and developed countries such as the United Kingdom and United States of America.

Many people who are given the bipolar disorder diagnosis prefer the original term, coined by Emil Kraepelin, of 'manic-depression' as this seems to reflect more closely the extremes of emotional experience they tend to report (Critical Psychiatry Network, 2009; Jamison, 1995). The term bipolar disorder was introduced by Karl Leonard in 1957 as a solution to the over-inclusiveness of 'manic-depression' due to the huge differences in the 'symptoms' reported by people given this diagnosis (Bentall, 2003). This separated manic-depression into unipolar depression and bipolar depression. Ironically, contemporary commentators observed that the construct of bipolar disorder has itself become an over-inclusive diagnosis in current diagnostic systems (Critical Psychiatry Network, 2009).

Critique of Psychiatric Diagnosis — In drawing from studies where diagnosis is part of the criteria for participant selection, it is important to acknowledge from the outset some of the problems of psychiatric diagnosis. Two of these problems are discussed below; lack of validity of diagnoses and ethical problems with diagnoses and its role in social power structures.

The psychiatric diagnostic system fundamentally views emotional distress as arising from biological abnormalities that can be alleviated by biological interventions (Moncrieff, 2007). Diagnostic systems have been argued to be unjustified by research and unreliable in distinguishing aetiology, prognosis and outcome (Boyle, 2007). This is perhaps not surprising given the historical context of diagnostic models and the fact that these have not been sufficiently updated to reflect the evidence of their invalidity (Bentall, 2003). Furthermore, it is clear that, despite service-users sometimes adopting diagnostic terminology to describe their experiences, they "tend to attribute their breakdowns not to illness, but to a variety of psychological and social reasons", taking a contradictory position to the disease model of diagnostic systems (Johnston, 2000, p. 35).

The diagnosis of bipolar disorder has received particular criticism. The Critical Psychiatry Network (2009) observes major discrepancies between the 'Diagnostic and Statistical Manual of Mental Disorders' and the 'International Statistical Classification of Diseases and Related Health Problems'. This lack of agreement between diagnostic systems undermines the validity of the construct of bipolar disorder. There has also been a concerning rise in reported prevalence rates of bipolar disorder since the 1950s and again more rapidly in recent years; this is difficult to account for using the disease model (Healy, 2008). One commentator has suggested that this reflects the investment of pharmaceutical companies in medication for bipolar disorder, which again would threaten the validity of the diagnosis (Healy, 2008).

In addition to validity concerns about diagnosis, there have also been questions about the oppressive function of diagnosis in society. Thomas Szasz (1972, p. 275) described psychiatric diagnoses as "stigmatizing labels, phrased 
to resemble diagnosis, and applied to persons whose behaviour offends or annoys others". This position has been further elaborated with some commentators perceiving a societal operation of power and interest in promoting a misinforming discourse of emotional distress as 'illness' that creates the illusion that the distress is the fault of the individual (Smail, 2005). Moreover, many service-users have described the burden and the stigma of diagnosis of mental illness as well as the subjugation of their views and opinions in professional discourses about diagnosis (Campbell, 2007).

Research Exploring This Diagnosis - Jones and Bentall (2006) argue that people who been given the diagnosis of bipolar disorder have until recently been neglected in psychological research. The recent upsurge in research has contributed to the development of theory and intervention. This has been based mainly on the integration of quantitative research that has explored personal factors related to risk, patterns of thinking, neuropsychological characteristics, biological characteristics, and treatment outcomes (Jones \& Bentall, 2006).

There is also a body of qualitative research that has sought to explore more closely the experience of people who have been given this diagnosis. These studies offer alternative insights into ways of understanding the experiences associated with symptoms and receiving diagnosis. The current impact of this qualitative research is limited due to the absence of attempts to make connections and draw distinctions between the findings of these studies.

\begin{abstract}
Aims
The purpose of this study is to review the current state of the literature reporting qualitative studies that depict to the experiences of 'symptoms' and 'having a diagnosis' for people who have been given a diagnosis of bipolar disorder. This consists of two questions; what are the experiences of 'symptoms' and 'having a diagnosis' for people who are given this diagnosis? What methods and theories have been used to explore this and how has this choice shaped the findings?

The undertaking of this study has inevitably been shaped from conception to completion by my own background, assumptions and beliefs. I am a male in my mid-twenties and my professional background is Clinical Psychology ${ }^{1}$. I am of Caucasian ethnicity and lived my whole life in England. Initially, my interest in this area arose from conversations with clinical colleagues about the difficulties of developing interventions for people who suffer from the kinds of distress associated with the diagnosis of bipolar disorder. Furthermore, in undertaking this study, I have become aware that part of my curiosity may reflect an interest in 'mania' due to personal experience of noticing, on occasion, my own feelings and actions taking on a 'hypomanic' quality. This reveals my own assumption that experiences described as 'mania' and 'depression' are ways of understanding different kinds of distress that are along a continuum of human experience as opposed to representing symptoms of an illness.
\end{abstract}

\title{
Method
}

\section{Meta-Review Methodology}

This meta-review uses an interpretive qualitative approach to compare and contrast qualitative research from the perspective of the insider. It is an attempt to combine analytical and discursive methods through a 'radical relativist' stance. This is a stance that rejects traditional notions of scientific 'truths' in favour of 'truth' as a social construction, whilst recognising the need to draw subtle links and connections between multiple perspectives of the 'truth' in order to make qualitative research useful (Sandelowski, 2006). 
Meta-Data Analysis - This is the "analysis of the results of previous analysis" (Zhao, 1991, p. 378), performed in order to highlight the interrelationships between findings regarding phenomenon under study (Paterson, Thorne, Canam, \& Jillings, 2001). In this manner, meta-data analysis is essentially an approach aimed at making sense of the findings of a series of related but independent investigations, in order to recognise shared and differing outcomes in a meaningful way. This meta-data analysis is guided by the meta-ethnographic approach of Noblit \& Hare (1988), which is described below.

Primary research studies identified for the literature review are read several times. They are then re-read and notes are made of the phrases, metaphors, ideas and concepts described in the primary studies. The next stage is to organise the notes from each study to identify common themes, metaphors and concepts within each study. Finally, the notes for all studies are compared to establish whether the studies offer similar ideas and concepts (demonstrating that they are 'reciprocal') or have conflicting ideas and concepts. Based on this process, the studies we analysed were perceived as being reciprocal and the shared themes that emerged are presented in this review.

Meta-Method - The meta-method provides an overview of the 'epistemological soundness' of the primary research studies (based on guidelines from Yardley, 2000), and a consideration of how the application of methods may have shaped the findings emerging from this field of research (Paterson et al., 2001).

Meta-Theory - The meta-theory undertaken in this review considers the theories that have been used to understand the experience of people who have a diagnosis of bipolar disorder and the theories developed from that body of research.

\section{Selection of Primary Research}

The initial searches attempted to identify any qualitative research papers that have studied the experience of people who have a diagnosis of Bipolar disorder. Studies were identified through a literature search of CSA PsychINFO, CSA PsychARTICLES, Science Direct (Elsevier), SwetsWise, Web of Knowledge, CINAHL and Medline. The following terms were used to locate the studies; 'bipolar' or 'bipolar disorder' or 'manic depression' or 'bipolar depression' or 'mania' AND 'qualitative' or 'Interpretative phenomenological analysis' or 'discourse analysis' or 'phenomenology' or 'discursive' or 'grounded theory' or 'narrative' or 'social constructions' or 'exploration' or 'focus group' or 'grounded study' or 'constant comparative'. In addition, the reference section of each of the studies was then explored to identify any further relevant studies.

Inclusion and Exclusion Criteria - After the initial literature search, more stringent inclusion and exclusion criteria were applied to select appropriate papers:

1. The research data must be based mainly on the accounts of the persons who are identified with a diagnosis of bipolar disorder.

2. The study must explore the person's experiences connected to their diagnostic label of bipolar disorder as opposed to understanding it in relation to a specific intervention or domain of life.

3. The study must be based on an adult population.

4. The study must have undertaken an analysis of the raw data.

5. The study must be physically or electronically accessible through reasonable measures to enable the authors to include it in the review. 
These criteria ensured that the studies relate to adults who have been given the diagnostic label of bipolar disorder. The choice of excluding studies without analysis of data was taken to provide a set of studies that also reveal something about the authors' constructions of participants' constructions. These strict criteria also provided a means for maintaining the number of studies below ten to enable this review to be comprehensive (Sandelowski, Docherty, \& Emden, 1997). Overall, nine studies were identified for inclusion in this review (see Table 1 below). For simplicity, this review refers to these studies by the code number in the table.

Table 1

Studies Identified for Review

\begin{tabular}{|c|c|}
\hline Code & 1 \\
\hline Study & Inder et al. (2008) \\
\hline Discipline & Psychiatry \\
\hline Country & New Zealand \\
\hline Data Collection & Therapy Sessions \\
\hline Analysis Method & Thematic Analysis (a form of interpretive theme analysis) \\
\hline Sample & 15 people with Primary Diagnosis of bipolar disorder (I, II, or 'not otherwise specified'). Age 15-35 \\
\hline Focus & The effect of bipolar disorder on the development of 'self'. \\
\hline Main Findings & $\begin{array}{l}\text { The authors identified four core themes in the experience of bipolar disorder; effects on relationships, others } \\
\text { definition of self, disrupting (impact of bipolar disorder on lives), and problems in the development of sense of } \\
\text { self. }\end{array}$ \\
\hline Code & 2 \\
\hline Study & Jönsson, Wijk, Skärsäter, \& Danielson (2008) \\
\hline Discipline & Nursing \\
\hline Country & Sweden \\
\hline Data Collection & Interview (semi-structured) \\
\hline Analysis Method & Content Analysis (a form of interpretive theme analysis) \\
\hline Sample & 18 People diagnosed with bipolar disorder (I or II). Age 18-57 \\
\hline Focus & How people with bipolar disorder understand their 'illness' and their view of the future \\
\hline Main Findings & $\begin{array}{l}\text { The authors identified six themes; accepting the illness, being insecure in oneself, striving for understanding, } \\
\text { managing the illness, an uncertain future and a hopeful future. The authors conclude this illustrates the ongoing } \\
\text { process of moving back and forth toward acceptance. }\end{array}$ \\
\hline Code & 3 \\
\hline Study & O'Leary, Page, \& Jenkins (1991) \\
\hline Discipline & Applied Psychology \\
\hline Country & United States \\
\hline Data Collection & Interview (semi-structured) \\
\hline Analysis Method & Phenomenal Analysis (a form of interpretive theme analysis) \\
\hline Sample & 4 people diagnosed with bipolar disorder "Manic Type." (Adults). Age not given \\
\hline Focus & What people experienced when they were in the 'mania stage' of bipolar disorder \\
\hline Main Findings & $\begin{array}{l}\text { Thirteen categories were identified. The authors discuss five themes in detail; levels of awareness, personal } \\
\text { behaviours, differences between manic phases, interpersonal behaviours and similarities between manic phases. }\end{array}$ \\
\hline Code & 4 \\
\hline Study & Proudfoot et al. (2009) \\
\hline Discipline & Psychiatry \\
\hline Country & Australia \\
\hline Data Collection & Email discussion between participant sample and informed supporters \\
\hline Analysis Method & Thematic Analysis (a form of interpretive theme analysis) \\
\hline Sample & 12 people diagnosed with bipolar disorder in 12 months prior to study and accessing treatment. Age 18-59 \\
\hline Focus & The experience, issues and concerns of people following diagnosis of bipolar disorder. \\
\hline
\end{tabular}


Main Findings

Code

Study

Discipline

Country

Data Collection

Analysis Method

Sample

Focus

Main Findings

\section{Code}

Study

Discipline

Country

Data Collection

Analysis Method

Sample

Focus

Main Findings

\section{Code}

Study

Discipline

Country

Data Collection

Analysis Method

Sample

Focus

Main Findings

\section{Code}

Study

Discipline

Country

Data Collection

Analysis Method

Sample

Focus

Main Findings

\section{Code}

Study
The authors identified seven themes; unwanted side-effects of medication, coping with unpleasant symptoms, positive and negative reactions to the diagnosis, identifying early warning signs and triggers of the illness, the loss of a sense of self, uncertainty about their future and stigma. They conclude these issues can undermine effective treatment and attempts at self-management.

\section{5}

Lim, Nathan, O'Brien-Malone, \& Williams (2004)

Clinical Psychology

Australia

Focus Group Discussion

The Phenomenological Approach (a form of interpretive theme analysis)

18 people with a bipolar I diagnosis. Age $28-58$

The psychosocial issues and problems faced by people given a bipolar disorder diagnosis.

Three themes were identified: bipolar patients' experience of life as chaotic and unstable, of circumstances as being out of their control, and as characterised by loss and deficits. The authors conclude that this may affect self-efficacy, which could negatively impact ability to manage the illness.

6

Michalak, Yatham, Kolesar, \& Lam (2006)

Psychiatry

Canada

Interviews (semi-structured)

Thematic Analysis (a form of interpretive theme analysis)

35 people with a diagnosis of bipolar disorder. Age 21-68

The impact of bipolar disorder on a person's life with specific reference to quality of life

The authors identified six themes; routine 'order in chaos', independence, stigma and disclosure, identity, social support, and spirituality. Authors also described participants' order of life priorities for quality of life contributing to the development of a quality of life questionnaire.

7

Goldberg (2007) $)^{2}$

Psychology (Clinical Emphasis)

United States

Interviews (Narrative)

Combination of grounded theory ideas (a form of interpretive theme analysis) with narrative \& discursive approaches to analysis

6 people who had a diagnosis of bipolar disorder. Age 40-55

Deconstructing the experience of people with a diagnosis of bipolar disorder

The author found an apparent mutual interaction between individual experience and societal labels. Participants had faced challenges to self and identity, with particular regard to developing a cohesive sense of self in the light of psychiatry and societal discourse about bipolar disorder.

\section{8}

Chapman (2002)

Educational Psychology

United States

Interviews (semi-structured)

Grounded Theory Analysis (a form of interpretive theme analysis)

12 people with a diagnosis of bipolar disorder (I and II). Age 22-62

The experience of bipolar disorder with a focus on responding to challenges to identity.

A central process in the experience of dealing with bipolar disorder is responding to challenges to identity. A model for understanding this process is developed and described.

9

Pollack \& Aponte (2001) 


$\begin{array}{ll}\text { Discipline } & \text { Psychiatric Nursing } \\ \text { Country } & \text { United States } \\ \text { Interviews (structured) } \\ \text { Analysis Method } & \text { Thematic Analysis (a form of interpretive theme analysis) } \\ \text { Sample } & 15 \text { people with a diagnosis of bipolar disorder on an inpatient ward. Age 21-52 } \\ \text { Focus } & \text { The perception of 'illness' in people with a diagnosis of bipolar disorder. } \\ \text { Main Findings } & \begin{array}{l}\text { The authors identified three themes; coming to terms with the diagnosis, the importance of personal metaphors, } \\ \text { and dealing with the medical model. There was no apparent difference between themes with regard to ethnic } \\ \text { groups. }\end{array}\end{array}$

Overall Participant Sample - All the studies included in this review used the official diagnosis of bipolar disorder as a way of selecting participants although the studies ranged widely in terms of the functional difficulties experienced by participants, the severity of their symptoms and the length of time since diagnosis. Overall, across all studies there were 135 participants. All studies, with the exception of one [5], stated the gender of participants and so overall data from these studies breaks down into the following percentages; $37.4 \%$ were men and $62.6 \%$ were women. The age of participants ranged from 15-68. Only four studies reported the average age of participants $[1,2,6,7]$. The mean of these average ages was 37.2 .

The ethnicity and education of participants was not widely reported in these studies. Given that bipolar disorder seems to be diagnosed with equal prevalence rates across different ethnic groups (Newman et al., 2001), it could be assumed that participants may represent largely the cultural majority within the countries where the studies took place. Therefore, this sample is likely to mainly represent white participants who live in the five specific countries where the research has been carried out, all of which would be considered to be 'developed' countries. The level of education of the participants indicates a range of educational backgrounds from school leave-takers before the age of sixteen to university graduates.

\section{Results}

\section{Meta-Data Analysis}

The meta-data analysis revealed observable and striking commonalities between the data of the studies. The steps we took to undertake this process are detailed in the methodology above. Nine major themes regarding the experience of 'symptoms' and having a diagnosis of bipolar disorder emerged. The quotations below are in bold for primary author's observations and italics for the participants' own words.

"...like a reed in the wind...I feel like I'm going like this in the breeze and l've lost whatever it says what I am" (1, p. 129).

All the studies with the exception of one [3] highlighted the struggles with identity faced by the participants. These struggles were described as complex and took multiple forms. Two of the studies [1,2] reflected on a sense of confusion that comes from the discontinuity of moods experienced by people who are given the diagnosis of bipolar disorder; "I had different personalities all over the place that were out of character for the person who had been so happy a couple of months ago" (1, p. 129). In some of the studies, the participants referred to a strong sense of loss or absence of self $[1,4,6,7,8]$. One participant said "my biggest issue is coming to terms with the loss of who I am, and that I sometimes feel I cannot be the person I once was" (4, p. 125) and another noted that after 
a manic episode, "You don't know how to become yourself again, it's like someone hands you a drivers licence and you're like, 'well who is this person'?" (6, p. 32).

Participants and authors in several of the studies also recognised a sense of fragility and uncertainty about the self $[1,4,7,8,9]$. The authors of one study noted that "Participants were not knowing whether or not to trust themselves" (4, p. 125), and a participant in another study described feeling like "...just a pile of pieces. And I didn't have enough glue to put it together again" (7, p. 155).

In contrast, four of the studies referred to 'acceptance' of identity as also being part of the experience of some people who have a diagnosis of bipolar disorder [1,2,6,8]. One author commented "As people sort through observations about self and symptoms, and as they consider what they value about themselves, they make decisions about what they will and will not claim as part of themselves" (8, p. 101).

"It's like walking down the road and having a big hailstorm coming" (8, p. 152).

Seven of the studies reflected on the feeling of loss of control as a central part of experience for participants $[1,3,4,5,7,8,9]$. Some of these studies commented that participants accounts of the internal experience of mania were partly characterised by a sense of fast motion and high speed thinking which felt out of control and scary $[3,4,7,8,9]$. This experience was powerfully communicated in the metaphors used by some of the participants. One person explained, "The mania terrifies me, the thoughts run through my mind like a speed train and I hate it." (4, p. 124). One study described the experience of "Very fast motion" as the only consistent characteristic of mania reported by participants $(3$, p. 45$)$.

In several of the studies, a frightening loss of control was also described by participants through the use of analogy to powerful and dramatic external events $[1,5,7,8]$. In particular, participants seemed to draw on metaphors from the natural world in communicating a sense of powerlessness in the face of overwhelming forces such as being in the path of hailstorms, tornados, quick sand and the waves of the ocean.

"The fear is that your mood might soar or that you might become depressed and unable to do what you have to...you don't dare to have long-term goals and dreams because you know that you are totally chaotic. You do not dare to dream." (2, p. 1229)

All of the studies in this review reported to some extent the participants' reflections of the experience of disruption in their lives and most studies highlighted a sense of pervasive uncertainty and instability that accompanied this in participants' accounts $[1,2,4,5,6,9]$. One study commented that, "There appears to be a constant struggle for stability and normality in their lives, and these are often elusive in the face of recurrent episodes and hospitalisation" (5, p. 813). This struggle for stability was described by the authors of another study as attempting to find "order in chaos" $(6$, p. 28).

Two studies commented directly on the shared experience among participants of uncertainty in terms of the future $[2,4]$. This uncertainty was seen as spanning all spheres of the person's life. A number of participants explained that as a result of this uncertainty, they avoid making plans and goals for the future, one example was "I only think about one day at a time...tomorrow I plan to go to my boyfriend...but then I haven't planned anything, it's like a blank piece of paper" (2, p. 1229). 
This uncertainty about the future was further allied to feelings of hopelessness in three of the studies $[2,4,5]$. One study hypothesised about the cognitive processes that lead to hopelessness, "If their future were predicted by their past and current circumstances, then it follows that they would perceive their future to be as uncertain or unstable as their lives at present. This often results in a sense of hopelessness..." $(5$, p. 815$)$.

“...saying inappropriate things would make me lose all my friends. And talking too fast, you know, all these things, acting weird, you know odd behaviour. My friends would ditch me of course and at the end l'd be alone" (6, p. 32).

Participants across all studies talked about the wide negative impact of symptoms on their social world and other domains of life. Two studies in particular highlighted this as a central part of the experience of their participants $[1,6]$. One of these described that, "Participant's responses revealed that the onset of bipolar disorder...brings about changes that, for many patients, may last a lifetime. These changes include the break-up of relationships, loss of employment and financial status, and alienation from friends" (6, p. 814). This interpretation by the authors also reveals their alignment to the medical model in assuming that 'bipolar disorder' is an illness that happens to a person and then causes life changes as opposed to there being an interaction between a person's distress and difficult life events. Furthermore, it is not clear in the authors' analysis whether this view is shared by the participants in the study.

All the studies revealed participant stories of deficits and losses with the theme of loss being made explicitly clear in four of the studies $[5,7,8,9]$. One participant explained "Our illness does cover loss. Loss, I mean, we have loss, living with loss, to do with relationships, jobs, and family, and also with time" (5, p. 814). In one study, the author observed that many participants had experienced feelings of loss as they, "began to realise that they have suffered significant losses in their own lives that, in retrospect, they can attribute to symptoms of their bipolar disorder" (8, p. 99).

In addition to experiencing loss about their current lives, two of the studies reflected on feelings of loss about the future $[8,9]$. These discourses contained a sense of grief and mourning for previously imagined futures now seen as unobtainable. One study summarised this loss as "not being able to fulfil their life dreams" [9, p. 175] and another extended this by observing that some of the participants realised "that, given their ongoing symptoms, their expectations and hopes for themselves are too high and need to be lowered to be more realistic" [8, p. 99).

"Thoroughly miserable week. I was having big issues about bipolar and how I hate the person I've become" (4, p. 126).

A common thread across five of the studies was a negative view of self $[1,4,5,8,9]$. Some participants expressed this directly through statements such as, "It can make you feel very, very bad about yourself, ...you can just feel like the lowest form of life on the planet" [8, p. 70]. Other's expressed a sense of self-disgust though their use of metaphor; "In the beginning I hid it like, like leprosy." (7, p. 202). For some of the participants it seemed that this sense of 'self-dislike' [1, p. 130] or "self-loathing" [1, p. 130] may have related to feeling like a failure, which was alluded to in two studies [1,5] with one observing that, "Bipolar patients also view themselves as being defective. Often, there is an accompanying sense of failure and loss of self-confidence" (5, p. 814).

It is interesting that this sense of failure was expressed only in two of the studies, which used non-interview based methods of data-collection, namely excerpts from therapy and a focus group. These methods may create a context 
where talking about a sense of self-failure may be more socially accepted or even socially expected than in interviews. This highlights the problem of comparing studies where different methods of data-collection are used, which place the participants in different social contexts and roles, such as interviewee, group member or serviceuser.

"Because somehow, the manic juice gives you somehow, the ability to make friends like mad. Great powers of conversation" (3, p. 45).

Four studies also described participants' accounts of positive elements of the experience of 'mania' $[2,3,4,7]$. For some, these positive elements of experience were seen by participants as offering relief from depression [2], whereas three of the studies [2,4,7] described how some participants found 'mania' a desirable part of experience in its own right; "Several of the participants commented on how they enjoyed their manic symptoms" (4, p. 124). One study developed this idea by eliciting a range of descriptions about the experience of 'mania' that indicated parts of this experience were positive, such as "... (feeling) very happy, feeling expansive, having high energy" [7, p. 113].

Participants in two of the studies expressed particular difficulty in taking on the assumption of the psychiatric construct of bipolar disorder, that would entail accepting that positive elements of their experience as part of 'an illness' $[4,7]$. One study commented, "They understood that while they were depressed they were unwell, but they found it harder to comprehend that when they were 'happy' they were also unwell" (4, p. 125). One study described the risk of 'pathologising exuberance' illustrated by one participant who said of getting diagnosed that, "Here's someone telling me that the times in my life where I had felt any joy, any ecstasy, any sense of purpose or meaning-you know...that any of those things that I felt or thought were also pathology" (7, p. 180).

"...you have to understand what a tremendous blow to my ego it was to be diagnosed with bipolar disorder...You cannot live the rest of your life like other people...the bottom line is, you're not NORMAL" (7, p. 133).

Participants in four of the studies described the significant impact of diagnosis and their subsequent responses and ways of finding meaning from this $[2,4,7,9]$. Some of the participants talked about difficulty initially accepting the assumption of the psychiatric model of the construct of bipolar disorder as 'an illness'; "Accepting my illness was very difficult for me...due to the fact that there were so many years between (relapses)" (2, p. 1223). One study indicated that part of the difficulty with accepting the diagnosis at first was the meaning of bipolar disorder to participants, "The initial association of some participants to bipolar disorder was that bipolar disorder was a 'crazy' diagnosis, unlike depression, and that this meant they were crazy" (7, p. 134).

The narrative offered within these studies seems to suggest that after the initial shock of diagnosis the participants went through a process of meaning-making that eventually led to the diagnosis becoming a more congruent part of their identity. This could be seen as a process of recognition that the construct of bipolar disorder closely resembled their experiences and matched their belief system, or alternatively the assimilation of diagnosis into their identity could be seen as enforced submission to assumptions of the psychiatric model due to the dominant influence of these discourses in society.

"I guess they just think about what they see on TV...They just assume...that you're crazy and you're nuts and you're psycho and you're dangerous" (6, p. 30). 
One of the strongest areas or reciprocity between the studies was participants' experience of feeling stigmatised $[1,2,4,5,6,7,8]$. Some of the participants' accounts seemed to indicate two parts to their experience of stigma; being seen as 'not normal' $[5,7]$ and being seen as 'crazy' or feared by other people $[5,6,7,8]$.

The sense of 'being seen as not normal' was illustrated in participants metaphors in one study, with one man describing feeling like a 'Martian', and another participant describing themselves as feeling like 'the kid with the cooties' [7]. The studies suggest that being seen as mentally ill and therefore not normal led to isolation from their communities. This discourse is summarised in one study, "They described being isolated from the community around them and their families, and view the community around them as rejecting them" $(5, p .814)$.

These studies also revealed narratives about the fears of being seen as crazy, with 'mania' being perceived as a particularly 'crazy' form of mental illness. This was captured by a participant in one study who said, "lt's sort of like it's ok to be depressed. It's not ok to be manic. Because, if you're manic, then they'll lock you up, or put a straight jacket on you, or something" (8, p. 63).

“...it's just something I grew from. Like each experience is all a piece of me, bad or good, and I do remember them and I do know what happened but there's nothing I can do about it and I know l've moved on and I can take that with me and it makes me stronger" $(1$, p. 130).

Another theme that seemed to be captured in some of the studies was a sense of acceptance and hopefulness $[1,2,6]$. The authors of the studies used phrases such as 'self-acceptance' (1, p. 130), 'personal growth' (2, p. 1227 ) and 'a hopeful future' (2, p. 1229) to describe this aspect of experience for participants. It was difficult to ascertain a consistent message from the studies about what acceptance involved. This could be due to the complicated and dynamic process of acceptance that was outlined by a participant in one study who described the process of acceptance as, "a whole bunch of different things (that) happen at different times, like physical acceptance of it, and the spiritual and emotional, and acceptance of the past and all the fears and things that go with it; it's quite a complex process" (6, p. 32).

\section{Meta-Method: A Methodological Critique}

Evaluating Epistemological Soundness - To assess the quality of these studies this review draws on the guidelines for good qualitative research developed by Yardley (2000). These consist of three guiding principles discussed below.

1. Sensitivity to Context - Almost all of the studies situated their research clearly within the context of previous literature and current theory $[1,2,3,4,5,7,8,9]$. Most of the studies prioritised positioning the research in the context of literature on bipolar disorder specifically, whereas one study drew on the wider context of literature regarding the experience of illness [9]. Two of the studies were able to place the research within the context of literature on bipolar disorder and wider understandings about the experience of diagnosis and people's experiences of 'illness' described in the field of health psychology $[7,8]$.

All of the studies with the exception of one provided a clear description of the recruitment procedures used [3]. The majority of studies used forms of convenience sampling, recruiting participants mainly from mental health services. Only one study reflected on how these recruitment procedures may have influenced the range of experiences reported by participants [7]. Two studies described an element of purposive sampling following initial recruitment with different intentions; one aimed to include participants who had covered issues pertinent to the re- 
search question at the initial phase of data collection [1], whereas another aimed to provide a heterogenous sample [6]. However, one study did use purposive sampling to provide an equal representation of ethnic groups [9].

Most of the studies gave some description of the participants' demographics, although a major limitation in many of the studies was an absence of contextual information such as ethnicity [1,2,3,4,5,6], gender [5], and level of education $[1,4,5,6]$. The relationship of the researcher in relation to the participants was rarely considered in these studies. It was not always clear if the participants had any existing working relationship with the researchers and there was no reflection on the impact of the relationship on the information that was elicited from participants.

One major limitation was that only one study provided a detailed description of the context of the researcher and how this may have influenced data-collection, data-analysis and subsequent findings [7]. In addition, the social role of the participants varied across these studies with some being interviewees $[2,3,6,7,8,9]$ and others being service-users in therapy [1], members of a focus group [5] or email respondents [4]. These different social roles may have influenced the way participants responded to researchers, which may reduce the validity of directly comparing these studies.

2. Commitment, Rigour, Transparency and Coherence - The notion of commitment as used by Yardley (2000) refers to prolonged engagement in the collection and analysis of data including a complete immersion in this data. The data collection stage was described with some clarity in all the studies. Some of the studies showed greater commitment and rigour in eliciting detailed data through the use of multiple interviews, contacts, or mediums of data collection $[1,4,5,7,8]$. Many of the studies used semi-structured or open interviews which took places over a period forty-five minutes of longer to ensure that participants had an opportunity to reflect extensively on the questions asked $[2,6,7,8]$. Three of the studies $[1,4,5]$ used innovative alternative methods for collecting data (excerpts from therapy sessions, email correspondents and focus groups).

Completeness of data-collection is an important part of undertaking rigorous research and this can be enhanced through the recruitment process (Yardley, 2000). This process was limited in this research as the majority did not describe the rationale for the number of participants with only two studies selecting participants on the basis of 'theoretical saturation' [7,8]. The completeness of data collection may also have been undermined through the use of strict exclusion criteria, including co-morbidity with other mental health difficulties [1], alcohol or drug dependence [1] or accessing services [1,4], high probability of disengaging with services [1], and currently being hospitalised [2].

The completeness of analysis and interpretation also contributes to rigour and this appeared to vary across studies. Most studies offered extensive and transparent evidence for the basis of analysis and interpretation through lengthy verbatim accounts that supported identified themes $(1,2,4,5,6,7,8)$, whereas one study used very specific participant quotes as evidence with little context of the overall account being given [3]. In contrast, one study provided few examples of evidence for each emerging theme, whilst using longer accounts directly from participants, giving a clearer flavour of the context and meaning of these accounts [9].

A strength of these studies was the appropriate fit between the research question and the underpinning philosophy of the methodologies used. All the studies drew on some form of interpretive theme analysis which matched the research questions adequately as this form of analysis seeks to identify aspects of the participants' experience 
from their own perspective, and in terms of their assumptions and constructs, which were seen as relatively stable over time (Dallos \& Vetere, 2005).

One study used mixed methods, drawing on three qualitative approaches: narrative analysis, interpretive theme analysis and discourse analysis, to identify themes, stories and discourses that run through participants' accounts [7]. Whilst the use of mixed methods can be criticised for lacking in coherence, this study was a good example of the important use of imagination and intuition in research (Cooper \& Stevenson, 1996) and appeared to offer a coherent way of understanding the data.

3. Impact and Importance - The studies in this review fulfilled the criteria of making an important contribution to understanding of the experience of people who have been given a diagnosis of bipolar disorder with potential for having a wide impact on clinical work. Whilst the qualitative methodology restricts the generalisability of the research conclusions, all authors attempted to consider the possible implications for clinical practice or future research.

This included contributing to the development of practical tools and methods for guiding clinical work or further research $[4,6,8]$, developing new theoretical understandings $[2,3,9,5]$, and raising specific issues for consideration in clinical work and research with people who have been given a diagnosis of bipolar disorder $[1,2,4,5,6,7,8,9]$. Two of the studies appear to have had a useful impact already as they have been part of research projects by the authors that were found to be widely cited as part of the literature review [6,9]. In contrast, two of the studies were dissertations projects, which at the time of our analysis had not been published and therefore would have at that point had little impact despite the potentially important contributions they offer to understanding the experience of people who have a diagnosis of bipolar disorder $[7,8]$.

Overall Appraisal of Primary Studies - As a whole, the studies reveal some interesting trends in the way the experience of bipolar disorder has been explored in qualitative research. It is notable that common themes emerged from research that span across five different countries. This may imply that there is a shared quality to the way in which different cultures socially construct and understand the psychological impact of being given a diagnosis of 'bipolar disorder'. This could reflect something regarding the 'international community of construction' aided by technological advances in the $21^{\text {st }}$ century. Alternatively, these commonalities could be perceived as supporting the notion that the participants' experiences reflect an 'actual phenomenon' that is less socially constructed and more inherent in 'human nature' itself.

One striking feature of the research is that all the studies were undertaken in developed and westernised countries. Furthermore, only one of the studies focused on a population whose first language was not English [2]. This may have contributed to the largely reciprocal nature of the concepts, metaphors and ideas expressed by authors and participants across studies. For example, the theme of 'struggles with identity' is perhaps a construction more consistent with the values and assumptions of westernised societies, and may not arise in research undertaken in more eastern cultures.

It is also notable that with the exception of one study, the research was all undertaken in the last decade. This may reflect an increasing popularity of qualitative methods or cultural shifts towards person-centred practice, where the personal narratives of service-users are given greater value than within previously dominant expertpractitioner models of working. All these studies reflected a sense of person-centred practice in their sensitivity and respect towards participants. However, most of the studies stopped short of stepping outside a medical model in taking the notion of 'bipolar disorder as an illness' at face value and using 'symptom-based' language. 
Furthermore, the participants were not active parts of the research process following data-collection, with the exception of one study that sought participant feedback on the themes that emerged [6].

Another important feature of this body of research is that in almost all the studies the chief investigator (first named author) was female $[1,3,4,5,6,7,8,9]$. Furthermore, this is coupled with a participant sample where males are underrepresented, given the equal prevalence of the application of a bipolar disorder diagnosis among the genders (Newman et al., 2001). It is possible that the paradigm of qualitative research fits more closely with dominant discourses available to women than the discourses available to men in western societies, which would lead both to women researchers being more interested in this method of study and women more likely to agree to participate in such research. The relative absence of male narratives in these studies may preclude developing understandings about how men who are given this diagnosis make sense of their experiences.

It is interesting that the majority of studies chose a semi-structured interview as the data-collection method. The popularity of this approach may reflect perceived best practice for undertaking good qualitative research, cultural traditions or practical issues. It could also be argued that interviews are only a tentative and small move away from the quantitative methodology of questionnaires.

Some of the primary research studies used more diverse and creative methods of data collection (such as therapy sessions and group forums), which raises a question about whether it is valid to have compared these differing studies in our analysis. It is my hope that including such a variety of studies can provide the foundation for a wider repertoire of discourses to be drawn on, which may enhance the helpfulness of this review, whilst at the same time it is important to acknowledge that this limits the validity and reliability of conclusions drawn from these studies as a whole.

Similarly, the studies also take different positions with regard to perceiving bipolar disorder as representing an 'illness' $[1,2,3,4,5,6,9]$ or viewing participants as a group of people who may have some shared experiences of distress that have contributed to them being labelled with the diagnosis of bipolar disorder [7,8]. On the one hand, this is problematic and undermines the cohesiveness of the findings but, on the other, it also perhaps usefully reflects the real world dilemma for practitioners who have to work alongside different models and find ways of communicating different ideas to service-users in respectful ways. One concern however, is that the majority of the studies do not even acknowledge they are taking a position within the disease model and as such create the illusion of an undisputed 'truth' that bipolar disorder is an illness.

The use of interpretive theme analysis in these studies may reflect a compromise in the research community between quantitative empirical research and qualitative methods due to using an approach that shares a quantitative assumption that individuals hold knowable, stable and robust assumptions and constructs that can be identified and assessed. One study used a more ambitious form of interpretive theme analysis [8], that of grounded theory, aiming to develop understandings that are generalisable to wider groups of people (Dallos \& Vetere, 2005).

\section{Meta-Theory}

Theoretical Perspectives Used to Understand the Phenomenon - The studies explored the experiences of people who have been given the diagnostic label of bipolar disorder through the lenses of several theoretical positions. Some of the studies drew mainly on one theoretical perspective $[1,3,5]$ for understanding these experiences, whereas others offered more eclectic perspectives $[6,7,8,9]$. All of the studies with the exception of one [3] explored 
the experiences of participants drawing on social psychology ideas. This seems consistent with the focus of participants on the connection between their experiences and their social relationships.

It is also possible however that this theoretical position may have impacted the framing and execution of the design, analysis and interpretation of findings towards an account convergent with social and psychological theories. In contrast, as discussed above, many of the studies were based firmly within a psychiatric 'illness' model of bipolar disorder that could be argued to be partly in contradiction with some of the social and psychological theories used to explain the findings.

The application of social theory was clearly evident in these studies. In one study, the author took a clear position of social constructionism from the outset [7] and in others the authors were influenced overtly by psychosocial theories [1,5] and by social ideas about the impact of stigma [2,4]. In other studies, the specific use of social theory was less explicit but remained present $[6,8,9]$. Two of the studies used specific psychosocial theories to explain their findings; Erikson's theory of identity diffusion in psychosocial development [1], and self-efficacy theory [5]. The absence of intrapsychic theories may account for the lack of explicit descriptions of emotional experience in these studies, becoming a subjugated discourse in the context of the more tangible social disruption experienced by the participants.

Three of the studies drew on theories derived from research into perceptions of illness and illness experiences in physical health practice $[4,8,9]$. This focus provided a useful comparison with similar literature that shared important experiential challenges with the accounts from participants in the studies, such as the experience of stigma and the challenge of identity. However, it could be argued that this reinforces the construction of these experiences as 'an illness' called 'bipolar disorder' that limits the authors' ways of understanding the participants' narratives.

Developments in Theory Derived From the Research - All the studies in this review exercised caution in making extensive theoretical claims on the basis of their findings. Instead, the studies opted to describe important themes that had been recognised by their participants, postulating that these may be useful to consider in clinical work or future research. However, some of the studies offered tentative theoretical developments on the basis of their findings.

One study applied self-efficacy theory (Bandura, 1995) to the understanding of the nature of bipolar disorder [5]. The authors reflected on ideas from self-efficacy theory in relation to their findings and concluded that a person's lack of belief in their ability to control 'moods' and 'symptoms' may influence their ability to develop self-management skills in order to help them manage their difficulties. The authors discuss implications for addressing self-efficacy beliefs in clinical work and measuring these beliefs as part of future outcome research.

One study attempted a more ambitious theoretical synthesis of its findings by proposing a new model for understanding the process of "responding to challenges to identity" [8]. The author suggests that an understanding of this model can enhance psychotherapeutic work with people who have a diagnosis of bipolar disorder.

\section{Discussion}

The accounts from authors and participants from the studies in this review illustrate the challenging journey of people who have been given a diagnosis of bipolar disorder. Some of the themes converge with quantitative research describing the 'symptoms' and characteristics of bipolar disorder. The theme of 'loss of control' shares 
similarities with the concept of 'psychomotor acceleration', which was one of five concepts identified as associated with manic of mixed symptoms in a study of people who have received a diagnosis of bipolar disorder (Cassidy, Forest, Murry, \& Carroll, 1998). In that study, they described 'psychomotor acceleration' as including 'symptoms' of increased motor activity, pressure of speech, flight of ideas and racing thoughts.

The same study identified a factor named 'increased hedonic function', which has parallels with 'positive or desirable aspects of mania' including increased humour, euphoria and increased interest in sexual activity. The presence of some positive aspects of mania is described further in autobiographies and single case accounts in academic journals (Furman \& Cavers, 2005; Jamison, 1995). However, all of these accounts also describe these desirable aspects of 'mania' in the context of the painful struggles experienced by people who have been given a diagnosis of bipolar disorder.

Similarly, the theme of 'negative view of self' is synonymous with findings from quantitative literature on the cognitive style of people who have a diagnosis of bipolar disorder. This literature suggests that people who receive a bipolar disorder diagnosis have low self-esteem and higher levels of self-criticism when 'depressed' or 'euthymic' than control groups of people who have not been given this diagnosis (Alloy et al., 2006).

In contrast, the remaining themes ('struggles with identity', 'disruption, uncertainty and instability', 'negative impact of symptoms across life and the experience of loss', 'struggling with the meaning of diagnosis', 'stigma', and 'acceptance and hope') are not well represented in the quantitative literature and therefore may be absent in discourses about working with people who have a diagnosis of bipolar disorder. This may be a consequence of the different focus of researchers using quantitative and qualitative approaches. The former are more likely to align themselves with the medical model, and explore issues of medication compliance and professional or expert perspectives on bipolar disorder, whereas the latter are perhaps more drawn to understanding the experience of participants and being curious about non-medical ways of describing these experiences.

However, some of the themes identified can lend themselves to be broadly applied to prevailing ideas about bipolar disorder recognised in the quantitative literature. The theme 'disruption, uncertainty and instability' is alluded to through causal models of bipolar disorder, which speculate on the role of disruption of biological and social experience in the 'relapse' of 'euthymic' people with a diagnosis of bipolar disorder (Luty, 2006). Similarly, the issue of non-adherence to treatment for people given this diagnosis is widely described in research (Gaudiano et al., 2008) and could relate to some of the identified themes, including 'struggles with identity', 'struggling with the meaning of diagnosis', and 'stigma'.

The theme of 'acceptance and hope' also appeared largely absent from the literature. This may reflect the tendency for clinical practice to focus almost entirely on the alleviation of suffering with little exploration of issues around positive wellbeing (Duckworth, Steen, \& Seligman, 2005). Attempts to explore these issues may begin to address problems such as treatment non-adherence, struggles with identity and diagnosis, stigma, and the negative view of self. If people who have been given a diagnosis of bipolar disorder are going to find new ways of being in the world that are less emotionally distressing, there needs to be a discourse about opportunities for developing salience, vitality and hope in their lives that offers a welcome alternative to flight into 'mania'.

\section{Implications for Clinical Practice}

The themes that emerged from the reviewed papers can be used in clinical work by practitioners through offering opportunities for people to explore these in therapeutic work. This may involve directly discussing the themes or 
simply thinking about how these issues may shape service-users' personal experiences and engagement with mental health services.

These themes can also inform the development and applications of specific interventions for people who have been given a diagnosis of bipolar disorder. 'Disruption, uncertainty and instability' and 'negative impact of symptoms across life and the experience of loss' can be addressed through the use of interpersonal social rhythm therapy which is aimed at developing a self-monitoring programme to facilitate a lifestyle, "characterised by more regular sleep-wake cycles, mealtimes, and other social zeitgebers” (Luty, 2006, p. 208).

Similarly, other practitioners are developing Mindfulness-Based Cognitive Therapy for bipolar disorder (Williams et al., 2008). This may offer new ways for people who have been given this diagnosis to live alongside, and be in acceptance of, 'disruption, uncertainty and instability', 'loss of control', and the 'positive or desirable aspects of mania', that could reduce the extent of resistance and judgement towards these experiences and therefore reduce the overall level of distress. Future clinical practice could consider even more radical use of the philosophies underpinning mindfulness by offering new narratives of identity that embrace the human capacity to experience the self as a collection of conflicting parts instead of aspiring towards the integration and wholeness goal of western philosophies (Epstein, 1998).

'Negative view of self' and 'loss of control' can be explored through the use of Cognitive Behavioural Therapy using techniques such as cognitive restructuring, positive datalogs and behavioural experiments (Mansell \& Scott, 2006; Newman et al., 2001). Newman et al. (2001) also discussed how therapists can work on the issues of stigma, loss and acceptance, which could offer effective ways of tackling these themes in individual work.

Two of the themes that emerged; 'stigma' and 'negative impact of symptoms on social relationships across life and the experience of loss' imply an important role for clinical work beyond that of individual approaches. In addition, the diagnosis itself is likely to contribute to the experience of stigma by placing the person's struggles entirely within the individual and presenting their distress as an illness. It could be argued that the most effective ways of tackling some of these difficulties is through challenging the use of illness terminology and diagnosis, raising public awareness and acceptance of people who are given this diagnosis and experience these kinds of distress, fighting discrimination and helping people to become and feel like a meaningful part of their community.

\section{Implications for Further Research}

The themes that were identified in the process of this review represent a collection of concepts, metaphors and ideas that communicate different understandings of the distress and unusual experiences reported by people who have been given the label of bipolar disorder. Future research could apply these themes using quantitative methods to explore their generalisability with larger numbers of participants. However, this relies upon this being a reliable and valid diagnostic category, and some have argued this is not the case with the diagnostic category of bipolar disorder (Bentall, 2003; Critical Psychiatry Network, 2009).

The qualitative research into the experience of people who are given this diagnosis could be extended by undertaking studies with greater male representation or with people from non-western countries. It would also be useful to explore the same experience using alternative methods than interpretive theme analysis, such as more purely discursive methods or narrative theme analysis. This would contribute wider perspectives on the experience of people given the diagnosis of bipolar disorder through recognising the importance of individual, local and societal contexts, discourses and narratives in shaping this experience. 
The identified themes also offer a variety of avenues for further elucidation and to explore in helping people suffering from similar kinds of distress to overcome these difficulties. One area where this could be particularly helpful is in exploring the theme of positive or desirable aspects of mania. The desire to retain these positive aspects of experience despite great suffering and distress may be undermining motivation to change and interventions to overcome these difficulties. This raises many questions about the nature, ideas and understandings about the experience of mania, pleasure, and happiness, for people who have been given a diagnosis of bipolar disorder and how this can be explored to make interventions more acceptable to service-users.

\section{Limitations of Review}

The specificity of the inclusion criteria may have limited the scope of this study to provide a comprehensive exploration of the experience of people who been given a diagnosis of bipolar disorder. The decision to exclude some studies on the basis of "understanding it in relation to a specific intervention or domain of life" inevitably relies on my own assumptions about what are important and central parts of experience for people given this diagnosis. As a result studies about 'identity' or 'psychosocial issues' were included, prioritising these parts of experience over that reported in excluded studies about 'smoking cessation' and 'work functioning'.

A possible limitation is the attempt to undertake both empirical and discursive analysis within the same study. This mixed methodology could be seen as being philosophically contradictory as the former assumes findings represent some form of reality and truth, whereas the latter analysis does not seek real truths but perspectives and ways of understanding. The stance of 'radical relativism' offered one way of resolving this issue where "Inquiry is pursued with truth as a 'regulated ideal'” (Sandelowski, 2006, p. 13). However, this has proved highly challenging and the discursive influence within this review may have itself been subjugated by my own tendency towards a stance of empiricism.

Another possible limitation, from an empirical perspective, is that this meta-review is inevitably based on my interpretations of the primary authors' interpretations (Paterson et al., 2001). This will have been influenced by the popular discourses available to me as a British, white, middle-class, male, in the field of Clinical Psychology. However, from a discursive perspective this layering of constructions is an inherent and unavoidable part of undertaking a qualitative review. However, tracking the origin of interpretations proved difficult in these studies. This may have diluted the authenticity of original experience as sometimes it was unclear which interpretations were direct descriptions from participants and which were reflections on a series of 'similar' accounts from participants but made by the authors. I have attempted to address this through being transparent about whether quotes have come from participants or authors, however this is limited as the review only has scope to present some of the core evidence for each theme from the synthesis.

\section{Notes}

1) This paper uses 'l' as a first person singular to represent specifically the subjective position of Leo Russell as the lead researcher.

2) Following the completion of this review, Goldberg's (2007) original dissertation has been published in an updated version of this work (Goldberg, 2012). 


\section{References}

Alloy, L. B., Abramson, L. Y., Neeren, A. M., Walshaw, P. D., Snezana, U., \& Nusslock, R. (2006). Psychosocial risk factors for bipolar disorder: Current and early environment and cognitive styles. In S. H. Jones \& R. P. Bentall (Eds.), The psychology of bipolar disorder: New developments and research strategies. Oxford: Oxford University Press.

American Psychiatric Association. (1994). Diagnostic and statistical manual of mental disorders: DSM-IV. Washington, DC: American Psychiatric Association.

Bandura, A. (1995). Self-efficacy in changing societies. New York: Cambridge University Press.

Bentall, R. P. (2003). Madness explained: Psychosis and human nature. Suffolk: Penguin Books.

Boyle, M. (2007). The problem with diagnosis. The Psychologist, 20(5), 290-292.

Campbell, P. (2007). Hearing my voice. The Psychologist, 20(5), 298-299.

Cassidy, F., Forest, K., Murry, E., \& Carroll, B. J. (1998). A factor analysis of the signs and symptoms of mania. Archives of General Psychiatry, 55, 27-32. doi:10.1001/archpsyc.55.1.27

Chapman, J. R. (2002). Bipolar disorder: Responding to challenges of identity (Doctoral dissertation). Retrieved from http://repositories.lib.utexas.edu/handle/2152/493

Cooper, N., \& Stevenson, C. (1996). Health research. In N. Cooper, C. Stevenson, \& G. Hale (Eds.), Integrating perspectives on health. Buckingham: Open University Press.

Critical Psychiatry Network. (2009). Comments on NICE bipolar guidelines: Second consultation. Retrieved from http://www.dbdouble.freeuk.com/Bipolar_2ndcons.htm

Dallos, R., \& Vetere, A. (2005). Researching psychotherapy and counselling. Buckingham: Open University Press.

Duckworth, A. L., Steen, T. A., \& Seligman, M. E. P. (2005). Positive psychology in clinical practice. Annual Review of Clinical Psychology, 1, 629-651. doi:10.1146/annurev.clinpsy.1.102803.144154

Epstein, M. (1998). Going to pieces without falling apart: A Buddhist perspective on wholeness. New York: Broadway.

Furman, R., \& Cavers, S. (2005). A narrative poem as a source of qualitative data. The Arts in Psychotherapy, 32, 313-317. doi:10.1016/j.aip.2005.02.004

Gaudiano, B. A., Weinstock, L. M., \& Miller, I. W. (2008). Improving treatment adherence in bipolar disorder: A review of current psychosocial treatment efficacy and recommendations for future treatment development. Behavior Modification, 32, 267-301. doi:10.1177/0145445507309023

Goldberg, S. G. (2007). The social construction of bipolar disorder: The interrelationship between societal and individual meanings (Doctoral dissertation). Santa Barbara, CA: Fielding Graduate University.

Goldberg, S. G. (2012). Becoming the denigrated other: Group relations perspectives on initial reactions to a bipolar disorder diagnosis. Frontiers in Psychology: Psychoanalysis and Neuropsychoanalysis, 3, Article 347.

Healy, D. (2008). Mania: A short history of bipolar disorder. Baltimore: John Hopkins University Press. 
Inder, M. L., Crowe, M. T., Moor, S., Luty, S. E., Carter, J. T., \& Joyce, P. R. (2008). "I actually don't know who I am": The impact of bipolar disorder on the development of self. Psychiatry, 71(2), 123-133.

Jamison, K. J. (1995). An unquiet mind: A memoir of moods and madness. London: Picador.

Johnston, L. (2000). Users and abusers of psychiatry. London: Routledge.

Jones, S. (2004). Psychotherapy of bipolar disorder: A review. Journal of Affective Disorders, 80, 101-114. doi:10.1016/S0165-0327(03)00111-3

Jones, S. H., \& Bentall, R. P. (2006) The psychology of bipolar disorder: New developments and research strategies. Oxford: Oxford University Press.

Jönsson, P. D., Wijk, H., Skärsäter, I., \& Danielson, E. (2008). Persons living with bipolar disorder: Their view of the illness and the future. Issues in Mental Health Nursing, 29, 1217-1236. doi:10.1080/01612840802370764

Lim, L., Nathan, P., O'Brien-Malone, A., \& Williams, S. (2004). A qualitative approach to identifying psychosocial issues faced by bipolar patients. The Journal of Nervous and Mental Disease, 192(12), 810-817. doi:10.1097/01.nmd.0000146734.39501.57

Luty, S. (2006). Biopsychosocial approaches and interpersonal and social rhythm therapy for bipolar disorder. In S. H. Jones \& R. P. Bentall (Eds.), The psychology of bipolar disorder: New developments and research strategies. Oxford: Oxford University Press.

Mansell, W., \& Scott, J. (2006). Dysfunctional beliefs in individuals with bipolar disorder. In S. H. Jones \& R. P. Bentall (Eds.), The psychology of bipolar disorder: New developments and research strategies. Oxford: Oxford University Press.

Michalak, E. E., Yatham, L. N., Kolesar, S., \& Lam, R. W. (2006). Bipolar disorder and quality of life: A patient-centred perspective. Quality of Life Research, 15, 25-37. doi:10.1007/s11136-005-0376-7

Moncrieff, J. (2007). Diagnosis and drug treatment. The Psychologist, 20(5), 296-297.

Newman, C. F., Leahy, R. L., Beck, A. T., Reilly-Harrington, N. A., \& Gyulai, L. (2001). Bipolar disorder: A cognitive behavioural approach. Washington: American Psychology Association.

Noblit, G. W., \& Hare, R. D. (1988). Meta-ethnography: Synthesizing qualitative studies. London: Sage.

O'Leary, E., Page, R. C., \& Jenkins, J. (1991). The awareness of mania as reported by bipolar persons. Psychology: A Journal of Human Behaviour, 27(4), 42-47.

Paterson, B. L., Thorne, S. E., Canam, C., \& Jillings, C. (2001). Meta-study of qualitative health research: A practical guide to meta-analysis and meta-synthesis. London: Sage.

Pollack, L. E., \& Aponte, M. (2001). Patients' perceptions of their bipolar illness in a public hospital setting. The Psychiatric Quarterly, 72(2), 167-179. doi:10.1023/A:1010371626859

Proudfoot, J. G., Parker, G. B., Benoit, M., Manicavasagar, V., Smith, M., \& Gayed, A. (2009). What happens after diagnosis? Understanding the experience of patients with newly-diagnosed bipolar disorder. Health Expectations, 12, 120-129. doi:10.1111/j.1369-7625.2009.00541.x 
Sandelowski, M. (2006). "Meta-jeopardy": The crisis of representation in qualitative metasynthesis. Nursing Outlook, 54(1), 10-16. doi:10.1016/j.outlook.2005.05.004

Sandelowski, M., Docherty, S., \& Emden, C. (1997). Qualitative metasynthesis: Issues and techniques. Research in Nursing \& Health, 20, 365-371. doi:10.1002/(SICI)1098-240X(199708)20:4<365::AID-NUR9>3.0.CO;2-E

Smail, D. (2005). Power, interest and psychology: Elements of a social materialist understanding of distress. Ross-on-Wye: PCCS Books.

Szasz, T. (1972). The myth of mental illness. Suffolk: Paladin.

Williams, J. M., Alatiq, Y., Crane, C., Barnhofer, T., Fennell, M. J., Duggan, D. S., . . Goodwin, G. M. (2008). Mindfulness-based cognitive therapy (MBCT) in bipolar disorder: Preliminary evaluation of immediate effects on between-episode functioning. Journal of Affective Disorders, 107, 275-279. doi:10.1016/j.jad.2007.08.022

Yardley, L. (2000). Dilemmas in qualitative health research. Psychology \& Health, 15, 215-228. doi:10.1080/08870440008400302

Zhao, S. (1991). Meta-theory, meta-method, meta-data-analysis: What, why, and how? Sociological Perspectives, 34(3), 377-390. doi:10.2307/1389517

\section{About the Authors}

Leo Russell is a Principal Clinical Psychologist working for Somerset Partnership NHS Foundation Trust in Adult Mental Health Services. His research interests include the experience of service-users given a diagnosis of bipolar disorder, working with males who suffer with eating difficulties, the use of positive psychology in the alleviation of emotional distress, and the clinical applications of intensive short-term dynamic psychotherapy.

Duncan Moss is a Senior Lecturer on the Clinical Psychology programme at the University of Plymouth, as well as a consultant and trainer in the areas of mindfulness meditation and reflective practice. His recent research interests have included a focus on exploring qualitatively the experience of 'mindfulness' meditation and looking at this in the context of broader reflective questioning of 'research' as discourse. 